\title{
Nucleic Acid Liquid Biopsies in Alzheimer's Disease: Current State, Challenges, and Opportunities
}

\author{
Tabea M. Soelter ${ }^{1}$, Jordan H. Whitlock ${ }^{1}$, Avery S. Williams ${ }^{1}$, Andrew A. Hardigan ${ }^{1,2}$, \\ Brittany N. Lasseigne ${ }^{\star 1}$
}

\section{Abstract}

Alzheimer's disease is the most common neurodegenerative disease and affects persons of all races, ethnic groups, and sexes. The disease is characterized by neuronal loss leading to cognitive decline and memory loss. There is no cure and the effectiveness of existing treatments is limited and depends on the time of diagnosis. The long prodromal period, during which patients' ability to live a normal life is not affected despite neuronal loss, often leads to a delayed diagnosis because it can be mistaken for normal aging of the brain. In order to make a substantial impact on AD patients, early diagnosis may provide a greater therapeutic window for future therapies to slow AD-associated neurodegeneration. Current gold standards for disease detection include magnetic resonance imaging and positron emission tomography scans, which visualize amyloid $\beta$ and phosphorylated tau depositions and aggregates. Liquid biopsies, already an active field of research in precision oncology, are hypothesized to provide early disease detection through minimally or non-invasive sample collection techniques. Liquid biopsies in Alzheimer's disease have been studied in cerebrospinal fluid, blood, ocular, oral, and olfactory fluids. However, most of the focus has been on blood and cerebrospinal fluid due to biomarker specificity and sensitivity attributed to the effects of the blood-brain barrier and inter-laboratory variation during sample collection. Many studies have identified amyloid $\beta$ and phosphorylated tau levels as putative biomarkers, however, advances in next-generation sequencing-based liquid biopsy methods have led to significant interest in identifying nucleic acids species associated with Alzheimer's disease from liquid tissues. Differences in cellfree RNAs and DNAs have been described as potential biomarkers for AD and hold the potential to affect disease diagnosis, treatment, and future research avenues.

\section{$\underline{\text { Keywords }}$}

Liquid biopsy, circulating biomarkers, Alzheimer's disease, neurodegeneration, cell-free, diagnosis

\footnotetext{
${ }^{1}$ Department of Cell, Developmental and Integrative Biology, The University of Alabama at Birmingham, AL, USA

${ }^{2}$ Department of Neurosurgery, Duke University Medical Center, Durham, NC, USA

* Correspondence: bnp0001@uab.edu
} 


\section{Background}

\section{Introduction}

Neurodegenerative diseases affect millions of patients worldwide, emphasizing the importance of early disease detection to increase the quality of life for patients and their families. Diseases caused by the degeneration of the nervous system portray a variety of phenotypes, including widespread memory loss and overall cognitive decline [1]. Parkinson's disease (PD), Amyotrophic lateral sclerosis (ALS), Alzheimer's disease $(A D)$, Vascular dementia, and Pick's disease are among the most prevalent neurodegenerative diseases, with AD being the most common type affecting persons of all races, ethnic groups, and sexes [1]. There are more than 40 million patients with AD worldwide and they can generally be categorized into early- and late-onset groups, defined by the age of onset of the disease [2]. Patients who develop symptoms before age 65 experience early-onset $A D$ (EOAD), accounting for roughly $5 \%$ of all AD cases [3]. Only 10-15\% of EOAD patients have an identified inherited autosomal dominant genetic pattern; therefore, the remaining cases are considered sporadic and have an unidentified cause [3]. Interestingly, individuals with identified genetic forms of EOAD often experience symptoms in their 30s and 40s in an extremely aggressive form of the disease compared to the lowprofile progression observed in late-onset AD (LOAD) patients [3]. Despite extensive research on AD progression and drug development, no cure or mitigating therapeutic has been approved since 2003, making successful AD treatments a continually unsatisfied demand [4,5].

Current diagnosis is based on clinical criteria which incorporate information on the patient's history, associated with memory loss and behavioral changes, accumulated through questionnaires of family members and others close to the patient [6]. Brain imaging technologies such as magnetic resonance imaging (MRI) and positron emission tomography (PET), which visualize protein depositions and aggregates in the brains of $A D$ patients, are utilized as supportive measures to increase the certainty of previous clinical diagnoses [6,7]. Despite being powerful tools and the current gold standard for AD diagnosis and detection, their inability to distinguish between different neurodegenerative disorders and the extreme monetary burden of these methods on patients and the healthcare system are considerable drawbacks [7].

Recent advances in liquid biopsy research in oncology have led to their clinical integration in the field of precision oncology, which has opened up the possibility of applying the same diagnostic tools to neurodegenerative diseases such as AD. However, a major hurdle in AD detection is the disease's long prodromal period, which coincides with the preclinical stage of the disorder, during which patients undergo small changes in their cognitive abilities resulting in minor effects on their ability to live a normal life [8]. Molecular diagnostics that allow for earlier diagnosis, evaluation of disease progression, and possible therapeutic monitoring are needed, as current diagnostic methods and interventions are often not employed until later in the disease course, greatly affecting patient outcomes. An alternative approach that adapts liquid biopsy protocols from oncology research may improve the detection of neurodegenerative disorders.

In order to implement liquid biopsy as a detection method in AD, potential biomarkers need to be identified. The brains of AD patients are characterized by disease hallmarks such as neuritic plaques caused by amyloid-beta aggregation, neurofibrillary tangles composed of hyperphosphorylated tau proteins, and neuroinflammation, which is thought to collectively lead to the development of dementia and cognitive impairment distinctive with $\operatorname{AD}[9,10]$. These disease hallmarks have been considered as biomarkers for liquid biopsies in $A D$, as well as various nucleic acid species. Nucleic acids leak from the brain into surrounding tissues such as the cerebrospinal fluid (CSF), which surrounds the brain and spinal cord, and into the bloodstream during aging $[11,12]$. While precision oncology commonly uses blood among other tissues for liquid biopsies, determining the most beneficial and least invasive tissue for AD detection is still being elucidated [13]. 
Challenges

AD's heterogeneous nature is at the foundation of many challenges accompanied with disease detection, biomarker identification, drug development and repurposing, and treatment. Different age phenotypes, genotypes, and etiology lead up to near-identical neuropathology of the disease, impeding the development of early detection methods and possible treatments [14]. Hence, elucidating a universal early disease detection biomarker panel for AD remains challenging. Multiple biofluids have been explored as potential candidates for liquid biopsies in neurodegenerative diseases with regard to collection, storage, and sensitivity. Despite being an ideal candidate for high specificity and sensitivity of AD biomarkers, CSF acquisition from patients is suboptimal. CSF collection methods are invasive and painful. The lumbar puncture, for example, requires the insertion of a large, hollow needle into the subarachnoid space for removal or collection of CSF [7]. Lumbar puncture requires a skilled operator and there are risks to the procedure including possible infection, bleeding and CSF leak with undesirable side effects such as headaches, dizziness, and nausea. Consequently, there would be significant challenges in the adoption of lumbar puncture for widespread diagnosis or screening efforts. [15].

These problems can be circumvented by utilizing blood collections, which can be performed repeatedly and are much less invasive. Additionally, aseptic blood collection protocols, processing, and proper temperature storage are commonly performed and are accessible nearly globally [16]. The World Health Organization (WHO) and others provide norms and standards for blood collection with room for further improvement [17-19]. A drawback of using blood for early disease detection is biomarker specificity and sensitivity, which is affected due to the low permeability of the blood-brain barrier (BBB), affecting biomarkers entering the bloodstream. The BBB is divided into multiple barriers primarily dependent on their location in the brain, all of which are affected by aging [11]. Over 20 research studies have implicated the breakdown of the BBB in samples for AD patients, before the onset of symptoms associated with $A D$ or mild cognitive impairment ( $\mathrm{MCl})$, a pre-dementia form of the disease [20]. This potential consequence may aid biomarker detection from blood collections. However, overcoming the BBB is crucial to increase the usefulness of blood for early detection of biomarkers originating in the brain, an avenue that is currently being pursued [21]. While CSF provides greater sensitivity and specificity to the central nervous system (CNS) nucleic acid biomarkers than blood, standardized protocols need to be implemented for biomarker analyses in blood and CSF. Especially CSF collection protocols lack standardization, greatly introducing variation to analyses of biomarkers [22]. In order to employ CSF to its full potential for liquid biopsies, there is a need for universal criteria and unified protocols to prevent inter-laboratory variation [23]. Despite their wide use, blood collection protocols require further adaptation for applications in circulating biomarkers.

A vital issue in $A D$ is the long prodromal period that precedes the onset of noticeable disease symptoms. It facilitates the disease's unimpeded progression due to a lack of early detection and treatment. Early detection would serve primarily as a neuroprotective measure, providing the opportunity to intervene early with the goal of stopping or slowing disease progression and ultimately increasing the patient's quality of life [24,25]. Repeatable blood collection and disease screening using liquid biomarkers could provide relief to patients who remain undiagnosed until the onset of severe symptoms. While there are no cures for AD, many drugs have been proposed and have undergone clinical trials, but $99.6 \%$ of AD clinical trials have failed [24]. A proposed cause for the high failure rate in $A D$ clinical trials is the late initiation of the study. Most studies begin after onset of $A D$ symptoms, which greatly affects outcomes since significant neuronal loss has occurred [24]. Therefore, early detection might also benefit drug trials and clinical studies by targeting the disease before it has progressed too far. However, initial symptoms of $\mathrm{MCl}$, which leads to $\mathrm{AD}$, are usually misidentified as typical aging, a problem that may be resolved through the implementation of liquid biopsies and their potential for early disease detection, which will eliminate false diagnoses [26].

Likewise, as most AD research heavily relies on the use of pre-clinical models, liquid biopsy can have an effect on the development of novel and more accurate models to study disease mechanisms, which are hard to 
observe in humans. AD mouse models are an alternative tool to study disease progression and have tremendously aided our understanding of the disease. Many models are for early-onset $A D$ and models accurately mimicking late-onset forms of the disease appear lacking [27]. Therefore, conclusions drawn from early-onset mouse models are often inaccurate in the context of LOAD because EOAD mouse models mimic inherited forms of $A D$ while neglecting to represent sporadic late-onset forms [28]. The underlying mechanisms of mouse neurodegenerative diseases may differ significantly from those in humans despite similar disease progression [29]. This differentiation further implicates the importance of creating predictive mouse models that accurately mirror human AD progression mechanisms. Liquid biopsies hold the potential to uncover underlying LOAD mechanisms at the preclinical stage, giving rise to the possibility to reorganize and reinvent $A D$ mouse models to include late-onset forms of $A D$ instead of being restricted to genetic EOAD.

Finally, technical and sequencing challenges associated with liquid biopsy also remain unaddressed. Next-generation sequencing (NGS) and droplet digital polymerase chain reaction (ddPCR) have previously been utilized to detect circulating tumor DNA from blood with high sensitivity in cancer [30]. ddPCR, an ultrasensitive targeted method, provides fast, inexpensive, and sensitive detection for cancer-specific mutations in liquid biopsy [31]. On the other hand, NGS methods for liquid biomarkers include targeted and untargeted detection approaches, which have the ability to perform massively parallel sequencing [31]. Unfortunately, targeted NGS methods, while showing high sensitivity, are limited by a predefined list of genes. Untargeted NGS methods are not limited to such predefined lists and are able to detect entirely novel changes at the cost of lower sensitivity, increased cost, and the necessity for higher input sample volume - which is particularly detrimental for samples like brain tissue or CSF, which are difficult to acquire [31]. Keeping these limitations in mind, cell-free DNA (cfDNA) detection methods require standardization in order to be introduced into the clinical setting [30,31]. Sequencing methods for small and long RNA-seq have been shown to create large inter-laboratory variation dependent on respective protocols. A study testing the accuracy and reproducibility of small RNA-seq library preparation methods was able to show decreases in inter-laboratory variation by 100 -fold through the implementation of standardized approaches [32]. Therefore, standardization of analytical and post-analytical factors, including experimental design, a sequencing protocol, quality control, and raw data processing is necessary to overcome current challenges associated with targeted and untargeted approaches in liquid biomarker research $[30,32]$. Despite being used in precision oncology, these methods are broadly applicable to $A D$, therefore addressing these limitations is a challenge for liquid biopsy studies in $A D$.

Despite some of the challenges with liquid biopsy development in $A D$, there remains great promise in their application: early disease detection, therapeutic monitoring, increased understanding of underlying disease mechanisms, and advances in pre-clinical models to include LOAD forms for future studies. These potential findings will advance our understanding of AD etiology, progression, and treatment with the goal of providing relief from the massive health burden caused by $A D$ and increasing patients' quality of life.

\section{$\underline{\text { Main Text }}$}

\section{Liquid Biopsy Sources and Tissues}

Several tissues have been examined as potential candidates for liquid biopsies in neurodegeneration, including AD. CSF has been shown to reflect biochemical changes in the CNS [7]. Despite its useful applications, obtaining CSF is invasive, requiring lumbar puncture resulting in significant discomfort and associated risks, as well as its limited availability hindering regular sample collection for repeated testing [7]. Conversely, blood collection is easily accessible and affordable, allowing for repeated collection without the side effects of pain and discomfort. This aids in the development of non-invasive pre-symptomatic screening protocols for AD despite concerns around its lower specificity and sensitivity for CNS biomarkers due to the BBB [7,33]. Finally, there have been reports of the potential usefulness of ocular, olfactory, and oral fluids for liquid biopsies. Multiple 
studies have shown differential ocular protein and miRNA levels between AD patients and healthy controls $[34,35]$. Koronyo and others highlight the practicality of detecting elevated protein levels of amyloid beta in the retina through imaging in $10 \mathrm{AD}$ patients and 6 healthy controls. At the same time, another study identified the elongation factor $4 \mathrm{E}$ in tear fluid of over half of the $\mathrm{AD}$ samples, which was absent in all controls, as well as an increase in total miRNA quantity in all 9 disease samples compared to $9 \mathrm{MCl}$ samples and 15 age-matched controls [34,35]. Significant increases in tau and phosphorylated tau protein levels were also detected from olfactory fluids of $A D$ patients, while controls did not exhibit such elevations [36]. Finally, Yilmaz and others described changes in concentration of 22 metabolites in oral fluids of patients with $\mathrm{MCl}$ and $\mathrm{AD}$ compared to healthy controls [37]. A significant advantage of the described fluids is their accessibility as well as their noninvasive collection procedures. However, the protein concentrations derived from these liquids are minimal, impacting their strength as a diagnostic tool for AD immensely, which will likely be resolved through further investigation [7,34-37]. Due to varying levels of accessibility of samples and specificity, studies reporting potential biomarkers in AD are often limited to blood and CSF collections.

\section{miRNAs}

Cellular damage, including injury to the brain tissue in $A D$, can lead to changes in the expression levels of miRNAs in the blood and CSF of patients with neurodegenerative diseases [38]. A major advantage of using miRNAs to detect changes in the biochemical processes of the brain is their ability to travel across the BBB in extracellular vesicles [39]. Additionally, miRNA molecules are detected in increased abundance and are more stable than higher molecular weight RNA molecules [40]. Serum-derived miRNA stability outperformed large RNA molecule stability in a multitude of conditions, including changes to $\mathrm{pH}$ levels, long-term storage, and increased freeze-thaw cycles [41,42]. Methods for miRNA profiling in liquid tissues include microarray analysis and next-generation sequencing approaches. However, progress has been limited by low circulating miRNA concentrations for which profiling methods usually require validation by RT-qPCR due to high miRNA variability, specifically in array analyses $[40,43,44]$. However, it is difficult to precisely identify the tissue of origin of detected miRNAs because amounts of extracellular vesicles (EVs), plasma lipoproteins, and proteins, as well as the type of miRNA extraction method performed, generate expression level biases of miRNAs that hinder tissue of origin identification [38].

Interestingly, miRNAs have been described as the most abundant nucleic acids in CSF and brain-derived extracellular fluid in patients with $A D$ and significant expression upregulation of miRNA-9, miRNA-125b, miRNA146a, and miRNA-155 has been shown in post-mortem brain tissues of 6 AD and 6 controls through array analyses [45]. On the other hand, these results have not been uniformly validated, especially in miRNA-146a, which has also been described as downregulated in CSF and hippocampus [46]. One potential explanation for these varying results is patient age and $A D$ stage. For example, miRNA-146a expression seems to be upregulated in earlier stages and downregulated in later stages of the disease, further emphasizing $A D$ heterogeneity [46]. A study in rats investigated the role of miRNA-146a in inflammation of the brain and suggests its involvement in neuroinflammation after performing a Pearson correlation analysis that indicates its potential role as an inflammation indicator [47]. Another study indicated the importance of miRNA-4422 on the expression dysregulation of beta-secretase 1 (BACE1) and gamma-secretase activating protein (GSAP) genes, which have been described to influence $A \beta 42$ deposition in $A D$ [48]. While many different miRNAs have been indicated to be involved in the neuropathology of the disease, it is worth noting that miRNA-9, miRNA-125b, and miRNA146a contribution has been evaluated repeatedly and shown promising results for AD detection [45,46,49-51].

Additionally, many studies aim to develop diagnostic models using cutting-edge computational approaches, including statistical tests comparing age-matched healthy controls to AD samples to discover novel biomarkers. Sheinerman and others used serum samples from patients with various neurodegenerative diseases and age-matched controls to determine that statistical approaches like. NET statistical packages and correlation 
analyses were able to distinguish healthy from affected individuals as well as to differentiate between different neurodegenerative diseases using brain-enriched miRNAs [52]. Additionally, machine learning approaches successfully differentiated AD patients from controls based on the expression levels of 21 miRNAs (18 of which were significantly correlated with neurodegenerative disease pathology) in blood, further implicating their utility as early diagnostic markers [53]. Another group developed a database of miRNAs differentially expressed in blood-related fluids and CSF of neurodegenerative diseases to recognize miRNA profiles specific to particular neurodegenerative diseases. This global miRNA study describes the intrinsic relationship between miRNAs and neurodegenerative diseases using bioinformatic approaches to identify therapeutic and diagnostic targets of miRNAs in neurodegenerative diseases [54]. Provided that findings describing the potential of miRNAs to detect neurodegenerative diseases, specifically $A D$, are validated further experimentally and computationally, miRNAs have the potential to drive forward the development of early-detection diagnostic tools for AD.

\section{tRNAs}

In recent years tRNA-derived RNA fragments (tRFs) have become a focus in human health and disease studies, and it has been shown that these degradation products play regulatory roles in cancer and neurological disorders [55]. Deficiencies in tRNA metabolism and processing enzymes have been linked to neurodegenerative disease, specifically in ALS and PD [56,57]. Defects in the RNA kinase CLP1 result in impaired pre-tRNA cleavage, increasing oxidative stress on neuronal cells, which drives cell degeneration [5861]. Similar to miRNAs, tRNAs and tRFs have been shown to enter circulation and avoid depletion in blood and serum due to their association with circulating extracellular vesicles, thus implicating their utility as a reliable circulating biomarker for AD [62]. Additionally, the presence of tRNA derivatives like stable 5' tRNA halves has been confirmed in mouse serum despite the derivatives' lack of connection to extracellular vesicles [63]. Utilizing circulating tRNAs and their derived fragments in neurodegenerative diseases have been largely understudied, even though they exhibit implications linked to neuronal cell death due to oxidative stress. Therefore further studies need to be conducted in this space.

\section{IncRNAs}

Long non-coding RNAs (IncRNAs) are non-coding RNAs (ncRNAs) that consist of sequences of $>200$ nucleotides and lack open reading frames and protein-coding abilities [64,65]. Nonetheless, IncRNAs have been implicated in AD pathology by regulating mRNA stability and have been described repeatedly as differentially expressed in animal models and patients of AD [65]. Early clustering attempts by the GENCODE consortium revealed that about $40 \%$ of IncRNAs are highly expressed in the brain, further delineating its potential role in neuropathology and utility as a diagnostic tool for early detection of the disease [66]. Since then, IncRNAs have been found to be dysregulated in brain tissues of AD mouse models compared to healthy and age-matched controls and demonstrate differential expression within disease models in an age-dependent manner $[67,68]$. Attempts to characterize IncRNAs involved in disease neuropathology have repeatedly described the involvement of IncRNA BACE1 in AD $[69,70]$. BACE1 is a crucial regulator in amyloid beta production, a ratelimiting step in AD progression [51]. Interestingly, IncRNA BACE1 levels were highly upregulated in plasma of AD patients when compared to controls in multiple studies, while other IncRNAs did not show such significant alterations [51]. Although BACE1 holds potential as a diagnostic biomarker, some studies have also proposed its use as a therapeutic target through the systematic delivery of BACE1 siRNAs and its inhibitors [71-73]. Studies investigating IncRNAs as potential biomarkers are not limited to BACE1, as PCA3, HOTAIR, and 51A are also investigated, with 51A showing increased levels in AD patient plasma [73]. An RNA-seq study identified several IncRNAs that were co-expressed with common AD-related genes [74]. Additionally, another RNA-seq study in ALS also described IncRNA dysregulation, and similar implications in PD, further indicating IncRNA utility in $A D$ and other neurodegenerative diseases $[75,76]$. Overall, there is considerable evidence for the 
involvement of IncRNAs in neurodegenerative diseases, while further research is necessary to fully understand their utility as a disease biomarker.

\section{cfDNAs}

Recently, cfDNA has become increasingly important for disease screening and early detection because cfDNA is released into circulation and insinuates the presence of disease [77]. The field of oncology has implemented cfDNA techniques to accurately screen for various cancer types, an avenue less pursued in neurodegeneration [78]. Hence, cfDNA has become of particular interest as a biomarker for neurodegenerative diseases $[79,80]$. Neuronal cell death, a process common in AD progression, releases DNA fragments into the bloodstream, which are hypothesized to be useful as a biomarker [79,81]. A study on $\mathrm{MCl}$ indicates that circulating cfDNA holds the potential to be utilized as a biomarker for overall cognitive decline [82]. However, cfDNA levels have been shown to vary significantly between individuals [83]. If the variation is unrelated to the disease state and due to inconsistencies in sample collection, processing, or analysis, this is a potential limitation of this particular nucleic acid. Additionally, cell-free mitochondrial DNA (cf-mtDNA) has been pursued as a potential AD biomarker as well. Detected in both plasma and CSF, damaged mitochondrial DNA is highly stable with low variability, which is particularly useful for consistent analytical approaches to distinguish between $A D$ and non-AD [84]. Multiple studies have shown cf-mtDNA depletion in AD samples as a useful biomarker in neurodegenerative diseases, a phenomenon not observed in healthy controls $[84,85]$. Both studies showed low levels of cf-mtDNA in CSF of $A D$ and PD patients and proposed it as an early diagnostic biomarker for neurodegenerative diseases [84-86]. While the utility of cfDNA in neurodegenerative diseases is not yet wellunderstood, circulating biomarker research is quickly expanding to include nucleic acids such as cell-free DNA as an early detection biomarker.

\section{Conclusions}

Liquid biopsy has only recently been accepted as a detection method for $A D$ and other neurodegenerative diseases with significant development due to technical advancements and increased understanding of $A D$ neuropathology. Continued development is underway to expand the implementation and usefulness of the approach. Since most neurodegenerative diseases do not have a cure, the demand for early detection methods is especially high to slow disease progression and increase patients' quality of life. AD drug trials' sizable failure rates call for changes in the approaches taken to research and treat the disease, a problem for which liquid biopsy can provide relief by uncovering novel therapeutic targets for future trials [16]. As an innovative tool it holds the potential to overcome limitations of traditional methods by improving early disease detection and screening through less-invasive techniques [87].

While the field of oncology has made great strides towards the successful implementation of liquid biopsies, development in neurodegenerative diseases trails behind. Multiple liquids have been proposed for the detection of $A D$ biomarkers and while the use of neural-derived blood extracellular vesicles has been proposed as a reliable and robust method for $A D$ detection and treatment, it has not received the same attention as blood and CSF [25]. Similarly, the usefulness of oral, ocular, and olfactory fluids remains understudied. As the least invasive fluids for detecting $A D$, these fluids hold ample potential for liquid biopsies but require further studies to determine their true potential, especially in larger cohorts than currently available [88]. CSF and blood are among the most studied and well-understood liquids for AD detection. A major advantage of CSF is high biomarker specificity and sensitivity, but lumbar puncture is still considered an invasive technique, while blood collections are minimally invasive and have been standardized globally, yet specificity and sensitivity are often lower in this fluid. Furthermore, an increasing number of studies demonstrate the affiliation between CSF and blood-based biomarkers and have indicated the power and usefulness of the latter [16]. 
The rapidly increasing discovery of potential biomarkers underscores the urgency of developing comprehensive blood- and CSF-based biomarker panels. Numerous nucleic acids like cfDNAs and RNAs have been proposed for $A D$ as well as the hallmarks of the disease. Further standardization of liquid collections and downstream analyses will open new avenues for drug development and treatments, ultimately increasing the quality of life and relieving the global health burden of $A D$ and other neurodegenerative diseases.

\section{List of Abbreviations}

$\begin{array}{ll}\text { AD } & \text { Alzheimer's disease } \\ \text { EOAD } & \text { Early-onset Alzheimer's disease } \\ \text { LOAD } & \text { Late-onset Alzheimer's disease } \\ \text { MCI } & \text { Mild cognitive impairment } \\ \text { PD } & \text { Parkinson's disease } \\ \text { ALS } & \text { Amyotrophic lateral sclerosis } \\ \text { BBB } & \text { Blood brain barrier } \\ \text { cfDNA } & \text { cell-free DNA } \\ \text { CNS } & \text { Central nervous system } \\ \text { CSF } & \text { Cerebrospinal fluid } \\ \text { MRI } & \text { Magnetic resonance imaging } \\ \text { PET } & \text { Positron emission tomography } \\ \text { AUC } & \text { Area under the curve } \\ \text { cf-mtDNA } & \text { Cell-free mitochondrial DNA } \\ \text { tRF } & \text { tRNA-derived RNA fragment } \\ \text { WHO } & \text { World Health Organization } \\ \text { NGS } & \text { Next generation sequencing } \\ \text { ddPCRDroplet digital polymerase chain reaction }\end{array}$

\section{Declarations}

\section{Ethics approval and consent to participate}

Not applicable

\section{Consent for publication}

Not applicable

\section{Availability of data and materials}

Not applicable

\section{Competing interests}

The authors declare that they have no competing interests. 


\section{Funding}

This work was supported by NIH R00HG009678 (to BNL, TMS also supported), the UAB Lasseigne Lab StartUp funds (to BNL), and the UAB Blazer Fellowship (to TMS, JHW).

\section{Contributions}

T.M.S. performed the literature search and wrote the manuscript. J.H.W. and A.S.W. contributed to the literature search. All authors read, edited, and approved the final manuscript.

\section{Acknowledgements}

We would also like to thank the members of the Lasseigne Lab, specifically Anisha Haldar and Timothy C. Howton for valuable comments and feedback on this manuscript.

\section{References:}

1. Selkoe DJ, Lansbury PJ Jr. Alzheimer's Disease Is the Most Common Neurodegenerative Disorder. In: Basic Neurochemistry: Molecular, Cellular and Medical Aspects 6th edition. Lippincott-Raven; 1999.

2. Naqvi E. Alzheimer's Disease Statistics [Internet]. 2017 [cited 2021 Apr 18]. Available from: https://alzheimersnewstoday.com/alzheimers-disease-statistics/

3. Awada AA. Early and late-onset Alzheimer's disease: What are the differences? J Neurosci Rural Pract. $2015 \mathrm{Jul} ; 6(3): 455-6$.

4. Winblad B, Amouyel P, Andrieu S, Ballard C, Brayne C, Brodaty H, et al. Defeating Alzheimer's disease and other dementias: a priority for European science and society. Lancet Neurol [Internet]. 2016 Apr [cited 2020 Dec 3];15(5). Available from: https://pubmed.ncbi.nlm.nih.gov/26987701/

5. Cummings J, Lee G, Ritter A, Sabbagh M, Zhong K. Alzheimer's disease drug development pipeline: 2019. Alzheimer's \& Dementia : Translational Research \& Clinical Interventions. 2019;5:272.

6. McKhann GM, Knopman DS, Chertkow H, Hyman BT, Jack CR, Jr, et al. The diagnosis of dementia due to Alzheimer's disease: Recommendations from the National Institute on Aging-Alzheimer's Association workgroups on diagnostic guidelines for Alzheimer's disease. Alzheimers Dement. 2011 May;7(3):263.

7. Lee JC, Kim SJ, Hong S, Kim Y. Diagnosis of Alzheimer's disease utilizing amyloid and tau as fluid biomarkers. Exp Mol Med. 2019 May 9;51(5):1-10.

8. Preclinical, Prodromal, and Dementia Stages of Alzheimer's Disease - Practical Neurology. [cited 2020 Dec 3]; Available from: https://practicalneurology.com/index.php/articles/2019-june/preclinical-prodromaland-dementia-stages-ofalzheimers-disease

9. Blennow K, Zetterberg H, Fagan AM. Fluid biomarkers in Alzheimer disease. Cold Spring Harb Perspect Med [Internet]. 2012 Sep 1 [cited 2020 Dec 3];2(9). Available from:

https://pubmed.ncbi.nlm.nih.gov/22951438/

10. Masters CL, Selkoe DJ. Biochemistry of amyloid $\beta$-protein and amyloid deposits in Alzheimer disease. Cold Spring Harb Perspect Med [Internet]. 2012 Jun [cited 2020 Dec 3];2(6). Available from: https://pubmed.ncbi.nlm.nih.gov/22675658/

11. Banks WA, Reed MJ, Logsdon AF, Rhea EM, Erickson MA. Healthy aging and the blood-brain barrier. Nature Aging. 2021 Mar 15;1(3):243-54. 
12. Huo Z, Zhu Y, Yu L, Yang J, De Jager P, Bennett DA, et al. DNA methylation variability in Alzheimer's Disease. Neurobiol Aging. 2019 Apr;76:35.

13. NCI Dictionary of Cancer Terms [Internet]. 2011 [cited 2021 Apr 18]. Available from: https://www.cancer.gov/publications/dictionaries/cancer-terms

14. Devi G, Scheltens P. Heterogeneity of Alzheimer's disease: consequence for drug trials? Alzheimers Res Ther. 2018 Dec 19;10(1):122.

15. Lumbar puncture (spinal tap) [Internet]. 2020 [cited 2021 Mar 8]. Available from: https://www.mayoclinic.org/tests-procedures/lumbar-puncture/about/pac-20394631

16. Hampel H, Goetzl EJ, Kapogiannis D, Lista S, Vergallo A. Biomarker-Drug and Liquid Biopsy Codevelopment for Disease Staging and Targeted Therapy: Cornerstones for Alzheimer's Precision Medicine and Pharmacology. Front Pharmacol. 2019 Mar 29;10:310.

17. WHO | Norms and standards. 2018 Jan 8 [cited 2021 Apr 12]; Available from: http://www.who.int/bloodproducts/norms/en/

18. O'Bryant SE, Edwards M, Johnson L, Hall J, Villarreal AE, Britton GB, et al. A blood screening test for Alzheimer's disease. Alzheimers Dement. 2016 Jun 25;3:83-90.

19. O'Bryant SE, Gupta V, Henriksen K, Edwards M, Jeromin A, Lista S, et al. Guidelines for the standardization of preanalytic variables for blood-based biomarker studies in Alzheimer's disease research. Alzheimers Dement. 2015 May;11(5):549-60.

20. Montagne A, Zhao Z, Zlokovic BV. Alzheimer's disease: A matter of blood-brain barrier dysfunction? J Exp Med. 2017 Nov 6;214(11):3151-69.

21. Pacia CP, Zhu L, Yang Y, Yue Y, Nazeri A, Michael Gach H, et al. Feasibility and safety of focused ultrasound-enabled liquid biopsy in the brain of a porcine model. Sci Rep. 2020 May 4;10(1):7449.

22. Perret-Liaudet A, Dorey A, Tholance Y, Dumont B, Quadrio I. Pre-Analytical and Analytical Critical Factors Influencing the High Variability of the Concentrations Levels of Alzheimer Disease Biomarkers in Cerebral Spinal Fluid. In: Zerr I, editor. Understanding Alzheimer's Disease. Rijeka: IntechOpen; 2013.

23. Janelidze S, Stomrud E, Brix B, Hansson O. Towards a unified protocol for handling of CSF before $\beta$ amyloid measurements. Alzheimers Res Ther. 2019 Jul 19;11(1):1-12.

24. The Need for Early Detection and Treatment in Alzheimer's Disease. EBioMedicine. 2016 Jul;9:1-2.

25. Abdel-Haq H. The Potential of Liquid Biopsy of the Brain Using Blood Extracellular Vesicles: The First Step Toward Effective Neuroprotection Against Neurodegenerative Diseases. Mol Diagn Ther. 2020 Dec;24(6):703-13.

26. Judge D, Roberts J, Khandker R, Ambegaonkar B, Black CM. Physician Perceptions about the Barriers to Prompt Diagnosis of Mild Cognitive Impairment and Alzheimer's Disease. Int J Alzheimers Dis. 2019 May 21;2019:3637954.

27. Onos KD, Sukoff Rizzo SJ, Howell GR, Sasner M. Toward more predictive genetic mouse models of Alzheimer's disease. Brain Res Bull. 2016 Apr;122:1-11.

28. Foidl BM, Humpel C. Can mouse models mimic sporadic Alzheimer's disease? Neural Regeneration Res [Internet]. 2020 Mar [cited 2021 Apr 11];15(3). Available from: https://pubmed.ncbi.nlm.nih.gov/31571648/

29. Götz J, Bodea L-G, Goedert M. Rodent models for Alzheimer disease. Nat Rev Neurosci. 2018 
Oct;19(10):583-98.

30. Thakral D, Das N, Basnal A, Gupta R. Cell-free DNA for genomic profiling and minimal residual disease monitoring in Myeloma- are we there yet? Am J Blood Res. 2020;10(3):26.

31. Heidrich I, Ačkar L, Mossahebi MP, Pantel K. Liquid biopsies: Potential and challenges. International journal of cancer [Internet]. 2021 Feb 1 [cited 2021 Apr 22];148(3). Available from:

https://pubmed.ncbi.nlm.nih.gov/32683679/

32. Giraldez MD, Spengler RM, Etheridge A, Godoy PM, Barczak AJ, Srinivasan S, et al. Comprehensive multi-center assessment of small RNA-seq methods for quantitative miRNA profiling. Nat Biotechnol. 2018 Jul 16;36(8):746-57.

33. Zetterberg H, Burnham SC. Blood-based molecular biomarkers for Alzheimer's disease. Mol Brain. 2019 Mar 28;12(1):26.

34. Kenny A, Jiménez-Mateos EM, Zea-Sevilla MA, Rábano A, Gili-Manzanaro P, Prehn JHM, et al. Proteins and microRNAs are differentially expressed in tear fluid from patients with Alzheimer's disease. Sci Rep. 2019 Oct 28;9(1):15437.

35. Koronyo Y, Biggs D, Barron E, Boyer DS, Pearlman JA, Au WJ, et al. Retinal amyloid pathology and proof-of-concept imaging trial in Alzheimer's disease. JCl Insight [Internet]. 2017 Aug 17;2(16). Available from: http://dx.doi.org/10.1172/jci.insight.93621

36. Passali GC, Politi L, Crisanti A, Loglisci M, Anzivino R, Passali D. Tau Protein Detection in Anosmic Alzheimer's Disease Patient's Nasal Secretions. 2015 [cited 2020 Aug 27]; Available from: https://pubag.nal.usda.gov/catalog/4664958

37. Yilmaz A, Geddes T, Han B, Bahado-Singh RO, Wilson GD, Imam K, et al. Diagnostic Biomarkers of Alzheimer's Disease as Identified in Saliva using 1H NMR-Based Metabolomics. J Alzheimers Dis. 2017;58(2):355-9.

38. Moldovan L, Batte KE, Trgovcich J, Wisler J, Marsh CB, Piper M. Methodological challenges in utilizing miRNAs as circulating biomarkers. J Cell Mol Med. 2014 Mar;18(3):371-90.

39. Cho KHT, Xu B, Blenkiron C, Fraser M. Emerging Roles of miRNAs in Brain Development and Perinatal Brain Injury. Front Physiol. 2019 Mar 28;10:227.

40. Etheridge A, Gomes CPC, Pereira RW, Galas D, Wang K. The complexity, function and applications of RNA in circulation. Front Genet. 2013 Jun 18;4:115.

41. Chen X, Ba Y, Ma L, Cai X, Yin Y, Wang K, et al. Characterization of microRNAs in serum: a novel class of biomarkers for diagnosis of cancer and other diseases. Cell Res. 2008 Oct;18(10):997-1006.

42. Cortez MA, Bueso-Ramos C, Ferdin J, Lopez-Berestein G, Sood AK, Calin GA. MicroRNAs in body fluids-the mix of hormones and biomarkers. Nat Rev Clin Oncol. 2011 Jun 7;8(8):467-77.

43. Batistela MS, Josviak ND, Sulzbach CD, de Souza RLR. An overview of circulating cell-free microRNAs as putative biomarkers in Alzheimer's and Parkinson's Diseases. Int J Neurosci. 2017 Jun;127(6):547-58.

44. Sheinerman KS, Umansky SR. Circulating cell-free microRNA as biomarkers for screening, diagnosis and monitoring of neurodegenerative diseases and other neurologic pathologies. Front Cell Neurosci. 2013 Sep 10;7:150.

45. Alexandrov PN, Dua P, Hill JM, Bhattacharjee S, Zhao Y, Lukiw WJ. microRNA (miRNA) speciation in Alzheimer's disease (AD) cerebrospinal fluid (CSF) and extracellular fluid (ECF). Int J Biochem Mol Biol. 
2012 Dec 24;3(4):365-73.

46. Müller M, Kuiperij HB, Claassen JA, Küsters B, Verbeek MM. MicroRNAs in Alzheimer's disease: differential expression in hippocampus and cell-free cerebrospinal fluid. Neurobiol Aging. 2014 Jan;35(1):152-8.

47. Xie Y, Chu A, Feng Y, Chen L, Shao Y, Luo Q, et al. MicroRNA-146a: A Comprehensive Indicator of Inflammation and Oxidative Stress Status Induced in the Brain of Chronic T2DM Rats. Front Pharmacol. 2018 May 14;9:478.

48. Hajjri SN, Sadigh-Eteghad S, Mehrpour M, Moradi F, Shanehbandi D, Mehdizadeh M. Beta-AmyloidDependent miRNAs as Circulating Biomarkers in Alzheimer's Disease: a Preliminary Report. J Mol Neurosci. 2020 Jun 1;70(6):871-7.

49. Guo R, Fan G, Zhang J, Wu C, Du Y, Ye H, et al. A 9-microRNA Signature in Serum Serves as a Noninvasive Biomarker in Early Diagnosis of Alzheimer's Disease. J Alzheimers Dis. 2017;60(4):1365-77.

50. Hong H, Li Y, Su B. Identification of Circulating miR-125b as a Potential Biomarker of Alzheimer's Disease in APP/PS1 Transgenic Mouse. J Alzheimers Dis. 2017;59(4):1449-58.

51. Zhao Y, Zhang Y, Zhang L, Dong Y, Ji H, Shen L. The Potential Markers of Circulating microRNAs and long non-coding RNAs in Alzheimer's Disease. Aging Dis. 2019 Dec;10(6):1293-301.

52. Sheinerman KS, Toledo JB, Tsivinsky VG, Irwin D, Grossman M, Weintraub D, et al. Circulating brainenriched microRNAs as novel biomarkers for detection and differentiation of neurodegenerative diseases. Alzheimers Res Ther. 2017 Nov 9;9(1):89.

53. Ludwig N, Fehlmann T, Kern F, Gogol M, Maetzler W, Deutscher S, et al. Machine Learning to Detect Alzheimer's Disease from Circulating Non-coding RNAs. Genomics Proteomics Bioinformatics. 2019 Aug;17(4):430-40.

54. Brennan S, Keon M, Liu B, Su Z, Saksena NK. Panoramic Visualization of Circulating MicroRNAs Across Neurodegenerative Diseases in Humans. Mol Neurobiol. 2019 Nov;56(11):7380-407.

55. Fu Y, Lee I, Lee YS, Bao X. Small Non-coding Transfer RNA-Derived RNA Fragments (tRFs): Their Biogenesis, Function and Implication in Human Diseases. Genomics Inform. 2015 Dec;13(4):94-101.

56. Greenway MJ, Andersen PM, Russ C, Ennis S, Cashman S, Donaghy C, et al. ANG mutations segregate with familial and "sporadic" amyotrophic lateral sclerosis. Nat Genet. 2006 Apr;38(4):411-3.

57. van Es MA, Schelhaas HJ, van Vught PWJ, Ticozzi N, Andersen PM, Groen EJN, et al. Angiogenin variants in Parkinson disease and amyotrophic lateral sclerosis. Ann Neurol. 2011 Dec;70(6):964-73.

58. Hanada T, Weitzer S, Mair B, Bernreuther C, Wainger BJ, Ichida J, et al. CLP1 links tRNA metabolism to progressive motor-neuron loss. Nature. 2013 Mar 28;495(7442):474-80.

59. Karaca E, Weitzer S, Pehlivan D, Shiraishi H, Gogakos T, Hanada T, et al. Human CLP1 mutations alter tRNA biogenesis, affecting both peripheral and central nervous system function. Cell. $2014 \mathrm{Apr}$ 24;157(3):636-50.

60. Schaffer AE, Eggens VRC, Caglayan AO, Reuter MS, Scott E, Coufal NG, et al. CLP1 founder mutation links tRNA splicing and maturation to cerebellar development and neurodegeneration. Cell. $2014 \mathrm{Apr}$ 24;157(3):651-63.

61. Weitzer S, Hanada T, Penninger JM, Martinez J. CLP1 as a novel player in linking tRNA splicing to neurodegenerative disorders: CLP1 in linking tRNA splicing to neurodegenerative disorders. WIREs RNA. 
2015 Jan 20;6(1):47-63.

62. Mishima E, Inoue C, Saigusa D, Inoue R, Ito K, Suzuki Y, et al. Conformational change in transfer RNA is an early indicator of acute cellular damage. J Am Soc Nephrol. 2014 Oct;25(10):2316-26.

63. Dhahbi JM, Spindler SR, Atamna H, Yamakawa A, Boffelli D, Mote P, et al. 5' tRNA halves are present as abundant complexes in serum, concentrated in blood cells, and modulated by aging and calorie restriction. BMC Genomics. 2013 May 2;14:298.

64. Jiang X, Lei R, Ning Q. Circulating long noncoding RNAs as novel biomarkers of human diseases. Biomark Med. 2016 Jul;10(7):757-69.

65. Li D, Zhang J, Li X, Chen Y, Yu F, Liu Q. Insights into IncRNAs in Alzheimer's disease mechanisms. RNA Biol. 2020 Jul 14;1-11.

66. Derrien T, Johnson R, Bussotti G, Tanzer A, Djebali S, Tilgner H, et al. The GENCODE v7 catalog of human long noncoding RNAs: analysis of their gene structure, evolution, and expression. Genome Res. 2012 Sep;22(9):1775-89.

67. Fang M, Zhang P, Zhao Y, Liu X. Bioinformatics and co-expression network analysis of differentially expressed IncRNAs and mRNAs in hippocampus of APP/PS1 transgenic mice with Alzheimer disease. Am J Transl Res. 2017 Mar 15;9(3):1381-91.

68. Lee DY, Moon J, Lee S-T, Jung K-H, Park D-K, Yoo J-S, et al. Distinct Expression of Long Non-Coding RNAs in an Alzheimer's Disease Model. J Alzheimers Dis. 2015;45(3):837-49.

69. Feng L, Liao Y-T, He J-C, Xie C-L, Chen S-Y, Fan H-H, et al. Plasma long non-coding RNA BACE1 as a novel biomarker for diagnosis of Alzheimer disease. BMC Neurol. 2018 Jan 9;18(1):4.

70. He W, Chi S, Jin X, Lu J, Zheng W, Yan J, et al. Long Non-Coding RNA BACE1-AS Modulates IsofluraneInduced Neurotoxicity to Alzheimer's Disease Through Sponging miR-214-3p. Neurochem Res [Internet]. 2020 Jul 17; Available from: http://dx.doi.org/10.1007/s11064-020-03091-2

71. Wang P, Zheng X, Guo Q, Yang P, Pang X, Qian K, et al. Systemic delivery of BACE1 siRNA through neuron-targeted nanocomplexes for treatment of Alzheimer's disease. J Control Release. 2018 Jun 10;279:220-33.

72. Sakamoto K, Matsuki S, Matsuguma K, Yoshihara T, Uchida N, Azuma F, et al. BACE1 Inhibitor Lanabecestat (AZD3293) in a Phase 1 Study of Healthy Japanese Subjects: Pharmacokinetics and Effects on Plasma and Cerebrospinal Fluid A $\beta$ Peptides. J Clin Pharmacol [Internet]. 2017 Nov [cited 2021 Jan 16];57(11). Available from: https://pubmed.ncbi.nlm.nih.gov/28618005/

73. Doxtater K, Tripathi MK, Khan MM. Recent advances on the role of long non-coding RNAs in Alzheimer's disease. Neural Regeneration Res. 2020 Dec 1;15(12):2253.

74. Shi Y, Liu H, Yang C, Xu K, Cai Y, Wang Z, et al. Transcriptomic Analyses for Identification and Prioritization of Genes Associated With Alzheimer's Disease in Humans. Front Bioeng Biotechnol [Internet]. 2020 [cited 2021 Jan 16];8. Available from:

https://www.frontiersin.org/articles/10.3389/fbioe.2020.00031/pdf

75. Joilin G, Gray E, Thompson AG, Bobeva Y, Talbot K, Weishaupt J, et al. Identification of a potential noncoding RNA biomarker signature for amyotrophic lateral sclerosis. Brain Commun [Internet]. 2020 Jun 17 [cited 2021 Jan 16];2(1). Available from: https://academic.oup.com/braincomms/article$\mathrm{pdf} / 2 / 1 /$ fcaa053/33639448/fcaa053.pdf

76. Lv Q, Wang Z, Zhong Z, Huang W. Role of Long Noncoding RNAs in Parkinson's Disease: Putative 
Biomarkers and Therapeutic Targets. Parkinson's Disease [Internet]. 2020 Jun 12 [cited 2021 Jan 16];2020. Available from: https://www.hindawi.com/journals/pd/2020/5374307/

77. Drag MH, Kilpeläinen TO. Cell-free DNA and RNA-measurement and applications in clinical diagnostics with focus on metabolic disorders. Physiol Genomics [Internet]. 2021 Jan 1 [cited 2021 Apr 11];53(1). Available from: https://pubmed.ncbi.nlm.nih.gov/33346689/

78. Swarup V, Rajeswari MR. Circulating (cell-free) nucleic acids--a promising, non-invasive tool for early detection of several human diseases. FEBS Lett. 2007 Mar 6;581(5):795-9.

79. MacBean LF, Smith AR, Lunnon K. Exploring Beyond the DNA Sequence: A Review of Epigenomic Studies of DNA and Histone Modifications in Dementia. Curr Genet Med Rep. 2020 Sep 1;8(3):79-92.

80. Santamaria BA, Luquin IB, Corroza J, Miguel MS, Roldan M, Zueco S, et al. Liquid biopsy shows differences in cfDNA fragmentation pattern between AD patients and controls: Biomarkers (nonneuroimaging) / plasma/serum/urine biomarkers. Alzheimers Dement [Internet]. 2020 Dec;16(S4). Available from: https://onlinelibrary.wiley.com/doi/10.1002/alz.039748

81. Chiu K-P, Yu AL. Application of cell-free DNA sequencing in characterization of bloodborne microbes and the study of microbe-disease interactions. PeerJ. 2019 Aug 6;7:e7426.

82. Feger D, Nidadavolu L, Oh E, Abadir P, Gross A. Circulating Cell-Free DNA Is Associated With Cognitive Outcomes. Innov Aging. 2020 Dec 16;4(Supplement_1):518-518.

83. Konki M, Lindgren N, Kyläniemi M, Venho R, Laajala E, Ghimire B, et al. Plasma cell-free DNA methylation marks for episodic memory impairment: a pilot twin study. Sci Rep. 2020 Aug 25;10(1):14192.

84. Cervera-Carles L, Alcolea D, Estanga A, Ecay-Torres M, Izagirre A, Clerigué M, et al. Cerebrospinal fluid mitochondrial DNA in the Alzheimer's disease continuum. Neurobiol Aging. 2017 May;53:192.e1-192.e4.

85. Podlesniy P, Figueiro-Silva J, Llado A, Antonell A, Sanchez-Valle R, Alcolea D, et al. Low cerebrospinal fluid concentration of mitochondrial DNA in preclinical Alzheimer disease. Ann Neurol. 2013 Nov;74(5):655-68.

86. Pyle A, Brennan R, Kurzawa-Akanbi M, Yarnall A, Thouin A, Mollenhauer B, et al. Reduced cerebrospinal fluid mitochondrial DNA is a biomarker for early-stage Parkinson's disease. Ann Neurol. 2015 Dec;78(6):1000-4.

87. Wert KJ, Velez G, Kanchustambham VL, Shankar V, Evans LP, Sengillo JD, et al. Metabolite therapy guided by liquid biopsy proteomics delays retinal neurodegeneration. EBioMedicine [Internet]. 2020 Feb [cited 2021 Jan 15];52. Available from: https://pubmed.ncbi.nlm.nih.gov/32028070/

88. Paraskevaidi M, Allsop D, Karim S, Martin FL, Crean S. Diagnostic Biomarkers for Alzheimer's Disease Using Non-Invasive Specimens. J Clin Med Res [Internet]. 2020 Jun 1;9(6). Available from: http://dx.doi.org/10.3390/jcm9061673 